\title{
Transfer matrix approach to the disordered Ising model on hierarchical lattices
}

\author{
Danielle O. C. Santos, Edvaldo Nogueira, Jr., and Roberto F. S. Andrade \\ Instituto de Física, Universidade Federal da Bahia, CEP 40210-210 Salvador, Bahia, Brazil \\ (Received 13 December 2005; revised manuscript received 25 March 2006; published 4 May 2006)
}

\begin{abstract}
A disordered short-range Ising model on the diamond hierarchical lattice, where the magnetic coupling constants $J_{i j}= \pm 1$ occur with probabilities $p$ and $1-p$, is investigated within a transfer-matrix based framework. Results are obtained after the evaluation of a large number of independent samples, where each individual coupling constant is randomly chosen according to the given probability distribution. The iteration of an exact set of discrete maps leads to the values of thermodynamic functions until a large but finite generation. An approximate scheme is developed to extend the results for each individual sample to the thermodynamic limit. Thermodynamic functions are evaluated and the $(T, p)$ phase diagram is obtained on the basis of the behavior of the correlation length. The critical value $p_{N}$ for the emergence of magnetic ordering is found to lie on the Nishimori line, and a small reentrant effect is observed. The exponent $\nu$ is evaluated for several values of $p$. Exact $\log$-periodic oscillations, found when $p=1$, vanish rapidly as $p$ decreases.
\end{abstract}

DOI: $10.1103 /$ PhysRevB.73.174202

PACS number(s): 05.50.+q, 05.70.Fh, 75.10.Pq

\section{INTRODUCTION}

The investigation of disordered magnetic systems relies heavily on the use of numerical simulations to obtain quantitative information on their thermodynamic behavior. ${ }^{1-3}$ At the same time, the rather rare analytical results are mostly quite applicable, as they set up useful guidelines to a large number of new questions and problems that correspond to some specific physical situations. This is the case, for instance, with Nishimori's contribution to the investigation of random Ising systems on Euclidean lattices where the nearest-neighbor coupling constants $J_{i j}$ are described by the following probability distribution:

$$
\begin{gathered}
P\left(J_{i j}=1\right)=p, \\
P\left(J_{i j}=-1\right)=1-p .
\end{gathered}
$$

Nishimori's findings are related to the existence of a continuous analytical line, the so-called Nishimori line (NL), expressed by

$$
\exp (2 J / T)=\frac{p}{1-p}
$$

in the $(p, T)$ diagram, where several thermodynamic properties of the model, including the internal energy, can be analytically evaluated. ${ }^{4,5}$ This result has been extended by a conjecture on the form of the $(p, T)$ diagram for $T<T_{N}$, where $\left(p_{N}, T_{N}\right)$ represents the point defined by the intersection of the ferromagnetic-paramagnetic border $T_{c}=T_{c}(p)$ with the Nishimori line. According to this conjecture, the boundary between the two phases for $T<T_{N}$ consists of a perpendicular line dropping vertically from $\left(p_{N}, T_{N}\right)$ to $\left(p_{N}, 0\right) .6$ This has given rise to a large number of works that seek to establish both the validity of this conjecture ${ }^{7,8}$ as well as whether the behavior of similar systems on non-Euclidian lattices is also described by the NL. ${ }^{9}$

In the two-bidimensional case, all numerical evidence available so far suggests that the critical point $\left(p_{N}, T_{N}\right)$ is located on the Nishimori line. Regarding the confinement of magnetic ordering to the low-temperature region $T<T_{N}$ and $p>p_{N}$, several authors have pointed out the presence of a reentrance of the ferromagnetic phase in the $(p, T)$ diagram, with a nonordering region when $p>p_{N}$ and $T \rightarrow 0 .{ }^{10} \mathrm{Al}-$ though it is well established that the lower critical dimension $\left(d_{\text {low }}\right)$ for the presence of a spin-glass (SG) phase in a disordered Ising system defined by a symmetric distribution is bounded to the interval $2<d_{\text {low }}<3,{ }^{11,12}$ Nishimori's result brought renewed interest to understand the behavior of twodimensional models with nonsymmetric distributions. ${ }^{13}$

In this work, we use a transfer matrix method to investigate the properties of a disordered Ising system with a probability distribution of ferro- and antiferromagnetic interaction defined by Eq. (1) on a diamond hierarchical lattice (DHL), for which the graph fractal dimensions $d_{f}=2 .{ }^{14,15}$ Also, we explore the dependence of the critical exponent $\nu$ with $p$, so that it is possible to compare our results with those obtained in recent works. ${ }^{16}$ This and similar models, defined on several distinct Euclidean and hierarchical lattices, have been investigated in a series of works, where different techniques have been used, e.g., Monte Carlo methods, ${ }^{17-19}$ transfer matrix $(\mathrm{TM}),{ }^{16,20}$ and real-space renormalization group (RG). ${ }^{21-24}$

Working within the RG framework, Nobre ${ }^{9}$ addressed the question of the form of the $(p, T)$ diagram for a particular autodual hierarchical lattice with the same value $d_{f}=2$. His results, based on analysis for both zero and finite temperature, indicate the presence of a very slight reentrance, i.e., the ferromagnetic border drops from $\left(p_{N}, T_{N}\right)$ to $\left(p_{c}, 0\right)$, with $p_{c}$ $>p_{N}$. It is well known that hierarchical lattices with the same fractal dimension may lead to different critical properties, so that it is worthwhile to explore the behavior for other lattices, e.g., those where autoduality is not present. This becomes more relevant for the present problem due to the very small size of the reentrant region. Our results for finite temperatures below $T_{N}$ suggest the presence of a very small reentrance.

The rest of this paper is organized as follows. In Sec. II, we present the model and discuss the major features of the 


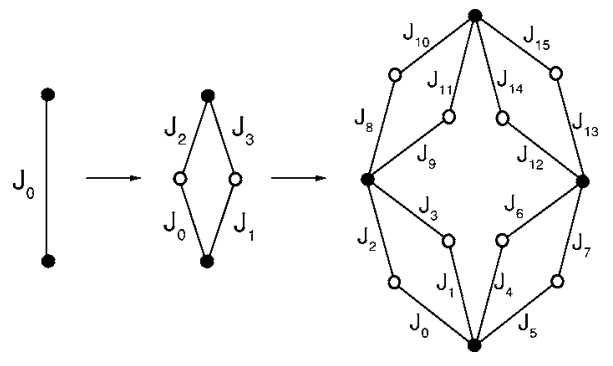

FIG. 1. First three steps of construction of a disordered model on a DHL. The constants $J_{k}$ are randomly chosen according to the PDF (1)

method used. Section III is divided into subsections. In the first one we discuss the behavior of some thermodynamic functions and the form of the $(p, T)$ phase diagram. In the latter, we present results for $\nu$ as a function of $p$, and we illustrate the rapid vanishing, for $p<1$, of the $\log$-periodic oscillations that are observed close to the para-ferromagnetic boundary when $p=1$. Section IV contains further remarks and conclusions.

\section{MODEL AND METHODS}

In this work we make use of a TM method that has been developed to investigate the properties of short-range periodic and aperiodic Ising models on DHL's. ${ }^{20}$ This approach has been recently adapted, with success, to the analysis of a fully disordered spin-glass model with symmetric distributions of ferro- and antiferromagnetic bonds. ${ }^{16}$ It amounts to direct evaluation of the thermodynamic properties for particular realizations of the model. The critical properties follow from the characterization of the singular behavior of the thermodynamical functions, particularly of the correlation length, averaged over a large number of samples.

The same strategy can be adopted at once to explore the asymmetric distribution probability of coupling constants given by Eq. (1). Its basic steps amount to starting with a formal Hamiltonian,

$$
H=-\sum_{(i j)} J_{i j} \sigma_{i} \sigma_{j},
$$

where $\sigma_{i}= \pm 1$ are Ising variables and the sum is carried over pairs of nearest neighbors on the DHL. The model is built with the lattice altogether, by replacing each bond of the previous generation $G$ by a set of two parallel branches, each with one inner site. If one starts with a single bond connecting the two root sites, this procedure gives rise to a selfsimilar graph, with fractal dimension $d_{f}=2$, and a nearestneighbor spin model mediated by coupling constants $\left\{J_{i j}\right\}$. The first steps of this procedure are illustrated in Fig. 1, where we explicitly indicate that the coupling constants $J_{i, j}$ are individually indicated, and do not follow any correlation to those placed between other pairs of spins. Note that, in Fig. 1, we have used a short-hand notation $J_{k}$ for the bonds $J_{i j}$, in order to avoid numbering the sites. For each realization, individual values for $\left\{J_{i j}\right\}$ are randomly assigned according to the probability distribution (1).
To obtain the thermodynamic properties of this model, the partition function for any DHL generation $G$ is written in terms of a $2 \times 2 \mathrm{TM} U_{G}$. This is accomplished by performing partial traces over all spins that are placed between the two root sites, $r_{1}$ and $r_{2}$. As the lattice is self-similar, partial trace at generation $G+1$ is expressed in terms of four independent partial traces of generation $G$. This can be formally put in terms of expressions that relate the eigenvalues $\eta$ and $\epsilon$ of two subsequent generations: $\eta_{G+1}=\eta_{G+1}\left(\eta_{G}, \epsilon_{G}\right)$ and $\epsilon_{G+1}$ $=\epsilon_{G+1}\left(\eta_{G}, \epsilon_{G}\right)$. To get rid of possible numerical overflows, it is most convenient to introduce a variable transformation, and define the free energy $f_{G}$ and correlation length $\xi_{G}$ by

$$
\begin{gathered}
f_{G}=-N_{G}^{-1} T \ln \eta_{G}, \\
\xi_{G}=\frac{M_{G}}{\ln \left(\eta_{G} / \epsilon_{G}\right)},
\end{gathered}
$$

where $N_{G}=2\left(2+4^{G}\right) / 3$ is the number of spins at generation $G$ and $M_{G}=2^{G}$ is the distance between the two root sites.

Then it is possible to rewrite the maps for $\eta_{G}$ and $\epsilon_{G}$ as $f_{G+1}=f_{G+1}\left(f_{G}\right)$ and $\xi_{G+1}=\xi_{G+1}\left(f_{G}, \xi_{G}\right)$. Such maps are explicitly written in Ref. 16, and we do not include them here for the sake of brevity. The only difference between the current implementation with respect to the previous one refers to the form of the probability distribution $P$, which is now given by Eq. (1).

This set of maps leads to the free energy and correlation length as functions of $T$ and the set $J_{i j}$ for successive values of $G$. If we let $G \rightarrow \infty$, these numerical values approach actual values in the thermodynamic limit. If we deal with homogeneous or deterministic aperiodic models, some 40-80 iterations are necessary to obtain $f(G)$ with a numerical precision of $10^{-16}$. The precise number depends on how close to $T_{c}$ is the value of $T$. We also observe that $f$ converges much faster than $\xi$. For any of the investigated situations, this represents no big challenge to usual desktop computers, as CPU time increases linearly with $G$.

In the disordered case, however, as we have to independently choose the coupling constants $J_{i j}$, the CPU time increases exponentially, and this constitutes severe burdens to the approach to the thermodynamic limit, especially when we focus on the behavior of $\xi$ and explore the region close to $T_{c}$. Then, besides the increased number of required iterations, we have to deal with strong fluctuations observed in the behavior of each individual sample with respect to sample average. In order to better appreciate the difficulties that are involved in the evaluation, a sample of 400 realizations for fixed $G=10$ (where each sample contains $\sim 10^{6}$ randomly chosen coupling constants) and a single value of $T$ requires some $10 \mathrm{~min}$ CPU time in an ordinary desktop computer with a $2.4 \mathrm{GHz}$ clock. This is enough to warrant a free energy relative dispersion of $10^{-2}$, relatively independent of the value of $T$. However, dispersion in $\xi$ remains at the same order of magnitude as $\xi$ itself, requiring a much larger value of $G(\sim 30)$ to reduce the fluctuations. As the CPU time increases with $4^{G}$, it seems to be hopeless, with the help of any available device to date, to obtain meaningful numerical results from the exact iteration of the maps. 
To sidestep this difficulty, we use again an approximate scheme, developed in Ref. 16. There we proceeded within an actual random choice of all coupling constants up to a given value $G_{M}$, and stored a large number of samples $N_{B}$ in a data bank. After this preliminary phase, we carried out iterations for larger values of $G$, in which we first built new $N_{B}$ samples at generation $G_{M}+1$, each one of them being formed by randomly choosing four samples of the data bank $G_{M}$. This scheme, which is repeated over and over while $G_{M}$ $<G \leqslant G_{\infty}$, amounts to a linear dependence of the CPU time with $G$, since no actually independent sample is constructed from the very beginning.

The idea of approaching the thermodynamic limit by working with a large set of finite-size samples is inspired in other approaches to spin glass systems, such as the renormalization group in hierarchical structures. ${ }^{12,21,24}$ There, banks of finite samples are successively generated from higherorder ones, and the critical point is obtained by the limit form of the probability distribution function as the number of decimation procedures is increased. The TM method used herein, when tested in the spin glass model, ${ }^{16}$ gave rise to reliable results both for the value of the critical temperatures and critical exponents.

Of course samples for $G>G_{M}$ start developing correlation, since the building blocks are always the $N_{B}$ samples at $G=G_{M}$. Despite this, the approximate scheme proved to be quite reliable, leading to results that are similar to those obtained by other approximative approaches.

\section{RESULTS}

\section{A. Thermodynamic properties and phase diagram}

Results obtained by the iteration of the maps for $f_{G}$ and $\xi_{G}$ can be displayed by average values $(\langle\cdot\rangle)$ of these quantities (or their derivatives), taken over a large number of independent samples, as a function of $T$, for fixed $p$. As we have discussed in the previous section, results for $f_{G_{M}}$ present good convergence already when we consider actual random systems up to $G_{M}=10$. From each $f_{G_{M}}$ it is possible to obtain also the entropy and specific heat $c$, as shown in Figs. 2(a) and 2(b), where we draw the corresponding curves for $p$ $=0.95$ and 0.97. Results for $\xi$ can also be obtained but, in the region close to the critical temperature, or even for the purpose of its better evaluation, the maps should be iterated for values of $G>G_{M}$ according to the scheme discussed before. This is also illustrated in Fig. 2(c), where we also draw $\left\langle\xi_{G_{M}}\right\rangle$ and $\left\langle\xi_{G_{\infty}}\right\rangle$, where $G_{\infty}=60$. The superposition of the curves indicates a matching of the numerical divergence of $\left\langle\xi_{G_{\infty}}\right\rangle$ with the cusp of $\langle c\rangle$ at $T_{c}$, while $\left\langle\xi_{G_{M}}\right\rangle$ remains finite in a region of $T<T_{c}$.

The behavior of the entropy with respect to the temperature is very smooth, and gives no hint of the possible existence of a critical point. Also, we notice the absence of residual entropy, as observed for the symmetric distribution $(p=0.5) .{ }^{16}$ Clearer evidence of critical behavior is provided by the presence of a cusp in the specific heat. However, Fig. 2(b) shows that, for smaller values of $p$, where the ferromagnetic ordering at low temperature becomes less defined by
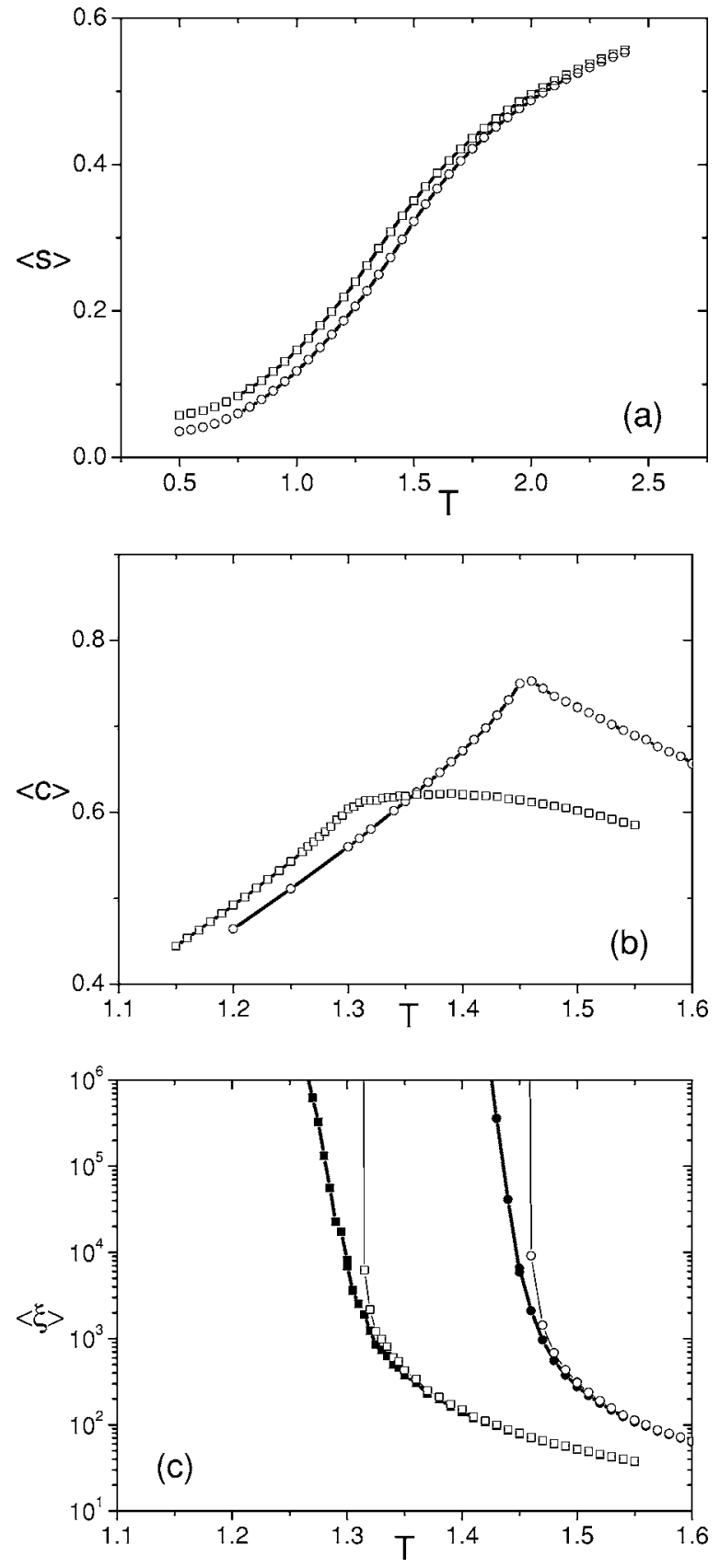

FIG. 2. Plots for $\langle s\rangle,\langle c\rangle$, and $\langle\xi\rangle$, for $p=0.95$ (squares) and 0.97 (circles). The largest value $G_{\infty}=60$. In (a) and (b), results for $G_{M}$ $=10$ and $G_{\infty}=60$ overlap for the used axis scale, and error bars are smaller than the height of the symbol height. In (c), results for $G_{M}$ (solid) and $G_{\infty}$ (hollow) become quite distinct as $T \rightarrow T_{c}$.

increasing bond disorder, the cusp of the specific heat becomes less and less pronounced. So, the identification of $T_{c}$ by the cusp position becomes unreliable. This is an expected phenomenon, as it is well known that transitions of disordered systems are not characterized by a critical behavior of $c$. Thus, when $p$ decreases, we are forced to use the divergence of $\xi$ to identify the value of $T_{c}$. For values of $p$ $\geqslant 0.97$, the dispersion from average values is very small, even $\xi$ in the region close to $T_{c}$. For the region of smaller $p$ values, the dispersion of $\xi$ increases when we approach $T_{c}$. In 


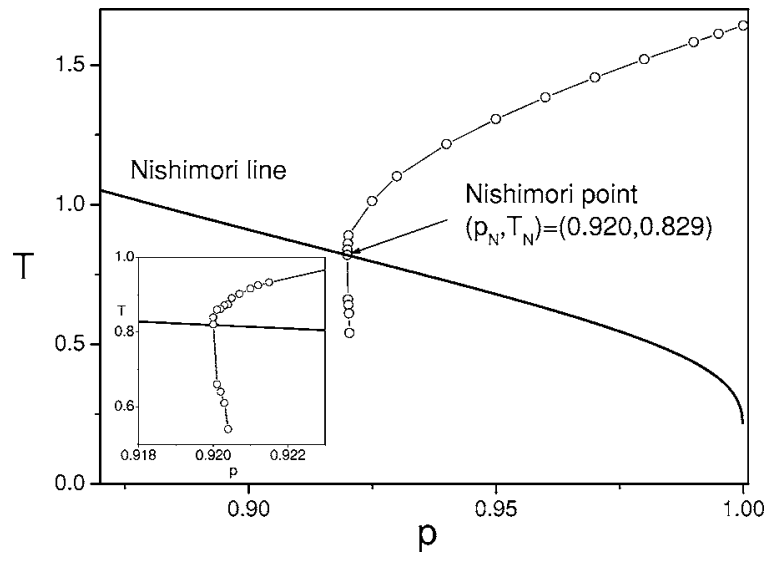

FIG. 3. $(p, T)$ phase diagram showing the boundary (circles and dashes) between the paramagnetic $(P)$ and ferromagnetic $(F)$ regions. Its intersection with the Nishimori line (solid) defines within $10^{-2}$ numerical accuracy the point $\left(p_{N}, T_{N}\right)$. The inset shows, for $T<T_{N}$, a small reentrant region.

this region, it is possible to have numerical divergence of $\xi$ for some samples, while for other samples, $\xi$ remains finite, albeit large. We locate $T_{c}$ by a simple criterion, taking into account the number of diverging samples: the paramagnetic phase persists until $\mathcal{N}_{d}(T)<\mathcal{N}_{s} / 2$, where $\mathcal{N}_{d}$ and $\mathcal{N}_{s}$ indicate, respectively, the number of diverging samples and the total number of samples in our investigation. For most of our investigation we have used $\mathcal{N}_{s}=400$.

The results for all values of $p$ and $T$ can be summarized in the diagram shown in Fig. 3. Phase transition from a paramagnetic state into an ordered phase is observed only for $p$ $\geqslant p_{N} \simeq 0.9200$, when $T_{c}\left(p_{N}\right) \equiv T_{N} \simeq 0.829$. We observe that this (Nishimori) point is located, within numerical precision, on Nishimori's line, which is also drawn in the graph.

As discussed before, the left border of the ordered region has been subject of a lengthy investigation, aiming to decide whether it falls down vertically from $\left(p_{N}, T_{N}\right)$ to $\left(p_{N}, 0\right)$ or it has a reentrant form so that, at $T=0$, the ordered phase is constrained to a region $p>p_{N}$. Our results, based on keeping track of the number of $\xi$ diverging samples, do support the existence of the reentrant region, as shown in the inset of Fig. 3. Note that, as obtained by other authors, this reentrant phenomenon is restricted to a very narrow $p$ interval close to $p_{N}{ }^{9}$ It is important to stress that this region is subject to very large fluctuation in the value of $\mathcal{N}_{d}$. Despite this, the presence of the reentrant region can be established with reliability, as illustrated in Fig. 4.

There we draw $\theta=\mathcal{N}_{d} / \mathcal{N}_{s}-1 / 2$ for $p=0.9200$ and $\mathcal{N}_{s}$ $=600$, as function of $T$, with increment $\Delta T=0.001$. Points corresponding to ferromagnetic ordering $(\theta>0)$ are widely intertwined with points where no ordering occurs $(\theta<0)$. To smooth the largely scattered data, we proceeded a moving window average $\langle\theta\rangle_{\text {mw5 }}$ taken over five successive values of $T$ (broken line). Though still quite irregular, it is possible to recognize a tendency toward having a region where $\langle\theta\rangle_{\mathrm{mw} 5}$ $>0$ in the central region of the $T$ interval. Finally, we have also evaluated a least-squares best fit with a fourth-degree polynomial $\langle\theta\rangle_{1 \mathrm{~s} 4}$ (solid curve). This curve shows very little sensitivity if it is evaluated working with the raw $\theta$ values or

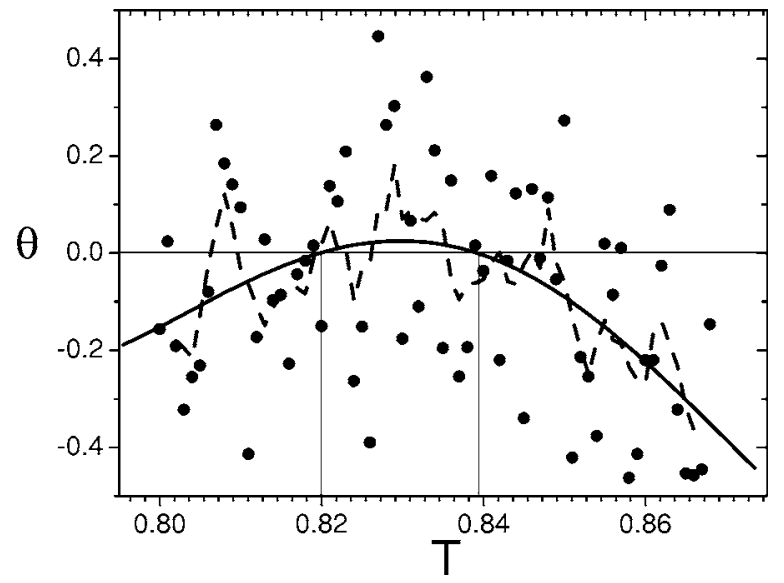

FIG. 4. Values of $\theta$ as a function of $T$, for $p=0.92$. The region corresponds to a small neighborhood of NP. The dashed line indicates $\langle\theta\rangle_{\mathrm{mw} 5}$ and the solid line the $\langle\theta\rangle_{\mathrm{ls} 4}>0$. The largest value of $\theta$ defined $T_{N} \simeq 0.829$.

with $\langle\theta\rangle_{\mathrm{mw} 5}$ values. This curves indicates a very narrow $\theta$ $>0 T$ interval $[0.820,0.839]$ where a ferromagnetic ordering is likely to occur. The largest value for $\theta$ locates NP at $T_{N}$ $=0.829$.

For $p=0.9199$, the same procedure no longer indicates a finite region where $\langle\theta\rangle_{\mathrm{ls} 4}>0$, while for $p=0.9201$ this interval is much larger than obtained for $p=0.92$, as we can observe from the inset of Fig. 3. This way we can locate within $10^{-4}$ and $10^{-2}$ precision, respectively, the values for $p_{N}$ and $T_{N}$. We also note that, for $p=0.92$, we obtain the corresponding value $T=0.819$ from NL analytical expression (2). This indicates that, to our numerical accuracy, the point $\left(p_{N}, T_{N}\right)$ of the DHL lies on NL.

Further, it is worth noting the asymmetric shape of the curve $\langle\theta\rangle_{\mathrm{ls} 4}$ with respect to its maximum. It decreases more rapidly for $T>0.839$ than for $T<0.820$, pointing to a sharper definition of the para-ferromagnetic boundary at larger temperatures. On the other hand, due to the very shallow reentrant region, points for $p=0.92$ and $T<0.82$ do feel the influence of a ordered phase in their close neighborhood, which can explain the slower decay of $\theta$.

Finally, similar analyses, with the same increment in $p$ and $T$, have confirmed a reentrant phase until $p \leqslant 0.9204$ and $T>0.54$. Our investigation has not been pushed to reach still lower values of $T$ because of the large values of the Boltzmann weights in this region. They bring to the numerical evaluation of $\xi$ a large number of intrinsic algorithmic instabilities leading to numerical overflows, which reduce the reliability of our results.

\section{B. Critical behavior}

The results of the thorough investigation of $\langle\xi\rangle$ as a function of $p$ and $T$, which were used to obtain the phase diagram, can also be explored to characterize the critical behavior of the system in the neighborhood of the paraferromagnetic boundary. So, let us now discuss results for the critical exponent $\nu$ in dependence of $p$. 


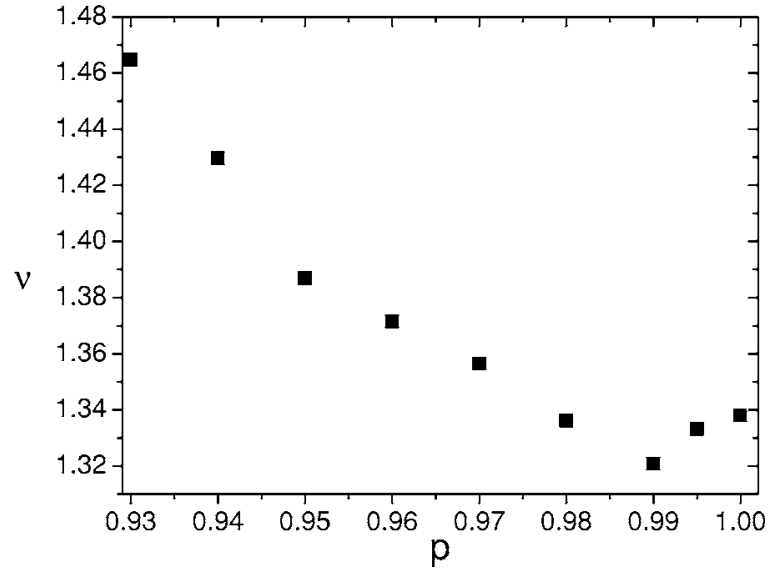

FIG. 5. Dependence of $\nu$ with respect to $p$.

First we recall that very precise values for $T_{c}$ have been obtained (within one part in $10^{5}$ ) for $p \geqslant 0.94$. Then, in order to evaluate $\nu$ with the same degree of accuracy, we reevaluated $\xi$ for several values $T_{i}>T_{c}$ so that $\log _{10} T_{i+1} / \log _{10} T_{i}$ $=\delta$ remains constant (typically $\delta=0.05$ ), and $t=\left(T_{c}-T\right) / T_{c}$ $\in\left[10^{-3}, 10^{-1}\right]$. With these data, it is straightforward to set up plots of $\xi$ as a function of the $t=\left(T-T_{c}\right) / T_{c}$ and proceed with the evaluation of $\nu$. The double logarithmic plots (not shown here) have a very regular linear dependence in the interval $\left[10^{-2}, 10^{-1}\right]$, even for $p=0.94$. For smaller values of $t$, strong fluctuations are present, so that the resulting pattern is that of a linear dependence (with roughly the same slope observed for the interval $\left[10^{-2}, 10^{-1}\right]$, superimposed to large fluctuations, the magnitude of which increase as $t$ and $p$ decrease.

The results for $\nu$ are shown in Fig. 5. We observe that, starting at the known value $\nu(p=1)=1.3382,{ }^{20}$ it first decreases with $p$, goes through a minimum for $p \simeq 0.99$, and then steadily increases as $p$ decreases down to $p=0.93$. For values of $p$ within a neighborhood $<0.01$, the presence of strong fluctuations, similar to those presented before, does not allow us to obtain values of $\nu$ with the same accuracy as displayed in Fig. 5.

The analysis of $\xi$ close to the transition line also makes it possible to follow how the presence of log-periodic oscillations evolves with $p$, as illustrated in Fig. 6. There we draw $d\left(\log _{10} \xi\right) / d\left(\log _{10} t\right)$ as a function of $\log _{10} t$ for $p=0.99$ and $\delta=0.0125$, so that the numerical derivatives become more accurate. Log-periodic oscillations are typical for models that have discrete scale invariance, such as the hierarchical lattices. ${ }^{20}$ For homogeneous or deterministically aperiodic magnetic models, very precise sinusoidal oscillations superimposed to a constant horizontal line that corresponds to the value $\nu$ are easily obtained. They contrast to the pattern shown in Fig. 6, where the quasirandom distribution of points over a straight horizontal line represents a dramatic change with respect to the quoted results.

Once the emergence of log-periodic oscillations is directly related to discrete scale invariance, we did expect that this behavior should disappear as $p$ decreases. However it is quite amazing to see that, at a still large distance from the Nishimori point, they are almost completely washed out.

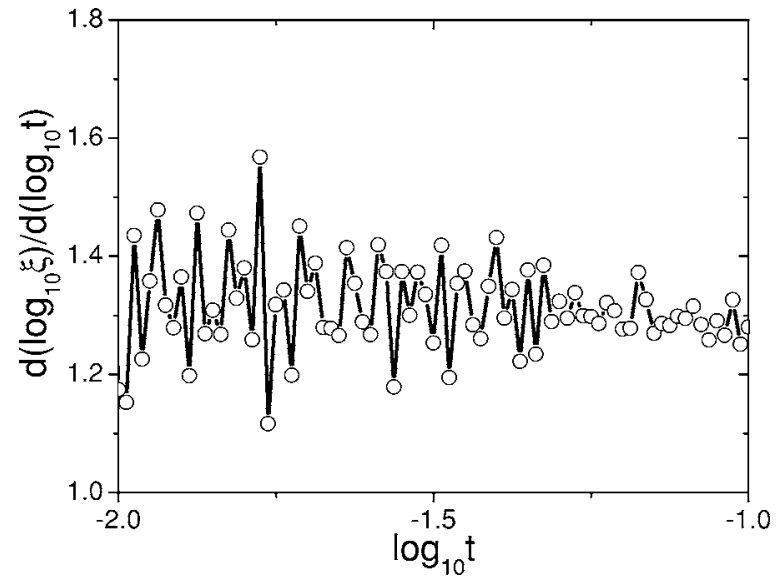

FIG. 6. Evidence of the rapid vanishing of log-periodic oscillations when $p=0.99$ deviates only slightly from $p=1$, where a wellformed sinusoidal pattern is found.

\section{CONCLUSIONS}

The results presented in this work have contributed to the understanding of the physical features of slightly perturbed magnetic systems. Working with the most simple diamond hierarchical lattice and a method based on the iteration of maps deduced from TM formalism, we constructed a phase diagram in the $p \times T$ plane, identifying the presence of ferromagnetic and paramagnetic regions. The Nishimori line plays an important role, as it accommodates the Nishimori point $\left(p_{N}, T_{N}\right)$, which sets lower bounds for the values of $p$ where ferromagnetic ordering can be observed. We have reported a very small reentrant boundary of the paramagnetic phase into the ferromagnetic one when $T<T_{N}$ and $p \gtrsim p_{N}$. This behavior is in accordance with those reported in several works on related models. ${ }^{9,23}$

Further, we have explicitly shown that the critical behavior depends strongly on the value of $p$, in accordance with some recent results reported in the literature. ${ }^{23}$ Starting from the previously reported value of the exponent $\nu$ when $p=1$, we first observed a slight decrease on its value when $p$ decreases, which is followed by a steady increase until $p_{N}$. We also investigated the effect of disorder on the emergence of log-periodic oscillations, which are observed for homogeneous and aperiodic models on hierarchical lattices. Our results show a very rapid decrease of log-periodic oscillations in dependence of $p$, indicating that, contrary to what occurs with aperiodic models, this pattern is very sensitive to the presence of random bonds. Finally, this second use of the TM scheme, developed in a previous work to describe thermodynamic properties of random systems on hierarchical structures, confirms its reliability and usefulness to treat problems that fit into this class.

\section{ACKNOWLEDGMENTS}

This work was partially supported by the Federal Brazilian granting agency $\mathrm{CNPq}$. The authors thank S. Coutinho for helpful discussions. 
${ }^{1}$ K. Binder and A. P. Young, Rev. Mod. Phys. 58, 801 (1986).

${ }^{2}$ K. H. Fischer and J. A. Hertz, Spin Glasses (Cambridge University Press, Cambridge, 1991).

${ }^{3}$ Spin Glasses and Random Fields, edited by A. P. Young (World Scientific, Singapore, 1998).

${ }^{4}$ H. Nishimori, J. Phys. C 13, 4071 (1980).

${ }^{5}$ H. Nishimori, Prog. Theor. Phys. 66, 1169 (1981).

${ }^{6}$ H. Nishimori, J. Phys. Soc. Jpn. 55, 3305 (1986).

${ }^{7}$ F. D. A. Aarão Reis, S. L. A. de Queiroz, and R. R. dos Santos, Phys. Rev. B 60, 6740 (1999).

${ }^{8}$ H. Kitatani and T. Oguchi, J. Phys. Soc. Jpn. 61, 227 (1992).

${ }^{9}$ Fernando D. Nobre, Phys. Rev. E 64, 046108 (2001).

${ }^{10}$ N. Kawashima and H. Rieger, Europhys. Lett. 39, 85 (1997).

${ }^{11}$ L. Klein, J. Adler, A. Aharony, A. B. Harris, and Y. Meir, Phys. Rev. B 43, 11249 (1991).

${ }^{12}$ E. Nogueira, Jr., S. Coutinho, F. D. Nobre, and E. M. F. Curado, Physica A 271, 125 (1999).
${ }^{13}$ H. Nishimori, Statistical Physics of Spin Glasses and Information Processing (Oxford University Press, Oxford, 2001).

${ }^{14}$ A. N. Berker and S. Ostlund, J. Phys. C 12, 4961 (1979).

${ }^{15}$ M. Kaufman and R. B. Griffiths, Phys. Rev. B 24, 496 (1981).

${ }^{16}$ R. F. S. Andrade, E. Nogueira, Jr., S. Coutinho, Phys. Rev. B 68, 104523 (2003).

${ }^{17}$ R. N. Bhatt and A. P. Young, Phys. Rev. Lett. 54, 924 (1985).

${ }^{18}$ A. T. Ogielski and I Morgenstern, Phys. Rev. Lett. 54, 928 (1985).

${ }^{19}$ Helmut G. Katzgraber, L. W. Lee, and I. A. Campbell, cond-mat/ 0510668 (unpublished).

${ }^{20}$ R. F. S. Andrade, Phys. Rev. E 61, 7196 (2000).

${ }^{21}$ B. W. Southern and A. P. Young, J. Phys. C 10, 2179 (1977).

${ }^{22}$ N. Kawashima and A. P. Young, Phys. Rev. B 53, R484 (1996).

${ }^{23}$ Michael Hinczewski and A. Nihat Berker, cond-mat/0507293 (unpublished).

${ }^{24}$ E. M. F. Curado and J. L. Meunier, Physica A 149, 164 (1988). 